Relations industrielles

Industrial Relations

\title{
Bibliography of Industrial Relations in the Railroad Industry, by James O. Morris, Ithaca, N.Y., New York State School of Industrial and Labor Relations, 1975, 153 p.
}

\section{Gérard Dion}

Volume 32, numéro 2, 1977

URI : https://id.erudit.org/iderudit/028792ar

DOI : https://doi.org/10.7202/028792ar

Aller au sommaire du numéro

Éditeur(s)

Département des relations industrielles de l'Université Laval

ISSN

0034-379X (imprimé)

1703-8138 (numérique)

Découvrir la revue

Citer ce compte rendu

Dion, G. (1977). Compte rendu de [Bibliography of Industrial Relations in the Railroad Industry, by James O. Morris, Ithaca, N.Y., New York State School of Industrial and Labor Relations, 1975, 153 p.] Relations industrielles / Industrial Relations, 32(2), 286-286. https://doi.org/10.7202/028792ar

Tous droits réservés @ Département des relations industrielles de l'Université Laval, 1977
Ce document est protégé par la loi sur le droit d'auteur. L’utilisation des services d’Érudit (y compris la reproduction) est assujettie à sa politique d'utilisation que vous pouvez consulter en ligne.

https://apropos.erudit.org/fr/usagers/politique-dutilisation/ 
négliger l'aspect administratif de la gestion des ressources humaines qui traite habituellement des objectifs et des politiques, du partage des responsabilités; et, plus précisément, de la gestion prévisionnelle des effectifs. Sans me faire le défenseur d'un enseignement théorique à outran$\mathrm{ce}$, je dois souligner que, même si tous les exercices sont pratiques et appropriés, ils ne sont pas suffisants pour permettre à l'étudiant ou à celui qui débute d'accéder à une vision cohérente et globale du domaine de la gestion des ressources humaines.

C'est une faiblesse qui peut être tournée en un avantage si l'on considère que le recueil laisse une grande marge de manœuvre au professeur qui tient à diffuser sa vision personnelle des choses et de recommander à ses étudiants les auteurs qu'il préfère ou encore qu'il connait bien.

Université Laval

Laurent BÉLANGER

Bibliography of Industrial Relations in the Railroad Industry, by James $\mathrm{O}$. Morris, Ithaca, N.Y., New York State School of Industrial and Labor Relations, 1975, $153 \mathrm{p}$.

Cet ouvrage est le douzième dans la série de bibliographie que publie la New York State School of Industrial and Labor Relations de l'Université Cornell. Il porte sur les relations industrielles dans l'industrie ferroviaire aux États-Unis.

Par «industrie ferroviaire», on entend le réseau des chemins de fer à l'exclusion du transport urbain et de la fabrication du matériel.

Le terme «relations industrielles" est pris ici dans son sens le plus large, c'està-dire que, loin de se limiter uniquement à la négociation collective ou aux rapports syndicaux-patronaux, il englobe tout ce qui se rattache aux relations professionnelles (emploi, chômage, syndicalisme, négociation, grève, médiation) ou qui les influence (législation, action gouvernementale, changements technologiques) ou qui, enfin, en est la conséquence (programmes de retraite, accidents du travail, etc.).

La bibliographie est divisée en chapitres selon six catégories de sources: a) bibliographie; b) les manuscrits; c) les livres, brochures et thèses; d) les périodiques; e) la documentation législative; 7) les publications gouvernementales. À l'intérieur de chacun des chapitres, la matière est ordonnée selon le plan suivant: généra- lités; les chemins de fer et le gouvernement ; le management ; la main-d'ceuvre; les conditions de travail; les syndicats et les travailleurs; la législation du travail ; les relations du travail ; les programmes de retraité et de protection des travailleurs.

La présentation a été effective en suivant le Manual of Style (12 édition) des Presses de l'Université de Chicago. Bien qu'il ne s'agisse pas d'une bibliographie annotée, lorsque cela s'avérait nécessaire quelques explications ont été ajoutées.

L'auteur nous avertit qu'il n'a pas la prétention d'offrir un ouvrage exhaustif sur le sujet mais, par ailleurs, que celui-ci dépasse largement ce que l'on qualifie habituellement de représentatif.

Naturellement cette bibliographie ne s'en tient qu'aux publications des ÉtatsUnis. Elle est cependant un instrument précieux et indispensable à tous les chercheurs qui auront à travailler sur cette question.

Université Laval

Gérard DION

Déontologie, Rapport du groupe consultatif de déontologie, Conseil des Arts du Canada, Ottawa, 1977, 36 pp.

Devant le nombre sans cesse croissant de demandes de subventions pour des recherches en sciences humaines mettant en cause «les intérêts et les préoccupations légitimes de sujets humains», le Conseil des Arts du Canada a formé un groupe de travail pour examiner les aspects éthiques de ce type de recherche et élaborer, si possible, un ensemble de règles de conduite à suivre en cette matière. Son rapport se présente sous la forme de réflexions générales sur la nécessité de l'obtention du consentement éclairé des sujets, le rejet catégorique du recours à toute forme de duperie ou d'artifices, le respect de l'intimité de ceux qui prêtent leur concours à une recherche ainsi que sur la confidentialité à leur garantir. Ces réflexions, qui sont assorties de recommandations précises, ont conduit le groupe de travail, composé d'universitaires d'origines diverses, à s'interroger sur les applications des principes généraux d'éthique à des situations particulières: acquisition et préservation de biens culturels, en anthropologie et en archéologie, recherches sur des populations «captives", sur les enfants, sur des cultures et groupes ethniques étrangers.

Les règles fondamentales que propose le Conseil des Arts aux chercheurs qui 